\title{
Einsamkeit bei Erwachsenen mit Sehbehinderung: Prävalenz, assoziierte Faktoren und Zusammenhang mit der Lebenszufriedenheit
}

\author{
Audun Brunes $^{\mathrm{a}}$ Marianne B. Hansen ${ }^{\mathrm{b}}$ Trond Heir ${ }^{\mathrm{a}, \mathrm{b}, \mathrm{c}}$ \\ a Section for Trauma, Catastrophes and Forced Migration - Adults and Elderly, Norwegian, Centre for Violence and Traumatic Stress

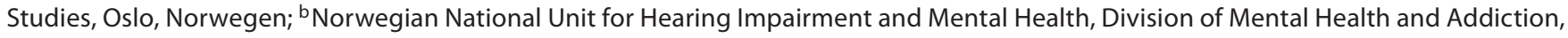 \\ Oslo University Hospital, Oslo, Norwegen; ' Institute of Clinical Medicine, Faculty of Medicine, University of Oslo, Oslo, Norwegen
}

\section{Schlüsselwörter}

Blindheit · Lebenszufriedenheit · Einsamkeit · Sehbehinderung

\begin{abstract}
Zusammenfassung
Hintergrund: Bisher ist wenig darüber bekannt, ob und in welchem Maße sich Einsamkeit auf das Leben von Menschen mit Sehbehinderung (SB) auswirkt. Das Ziel dieser Studie war es daher, die Prävalenz und die assoziierten Faktoren von Einsamkeit bei Erwachsenen mit SB sowie den Zusammenhang von Einsamkeit und Lebenszufriedenheit zu untersuchen.
\end{abstract}

Methoden: In diese Querschnittsstudie wurde eine Wahrscheinlichkeitsstichprobe von 736 Erwachsenen ( $\geq 18$ Jahre) mit SB eingeschlossen, die Mitglieder des Norwegischen Verbandes der Sehbehinderten und Blinden waren. Die Interviews fanden zwischen Januar und Mai 2017 statt; erhoben wurden Informationen über soziodemographische Faktoren, Merkmale der SB, negative Lebenserfahrungen, Einsamkeit laut Three Item Loneliness Scale und Lebenszufriedenheit laut Cantril's Ladder of Life Satisfaction. Die Prävalenz der Einsamkeit wurde mit Daten der norwegischen Allgemeinbevölkerung ( $N=14$ 884; Durchschnittsalter 46,4 Jahre; 50,7\% Frauen) verglichen.
Ergebnisse: Die Prävalenz mittelschwerer und schwerer Einsamkeit in der SB-Population betrug 28,7\% (95-\%-Konfidenzintervall (KI): 25,4; 32,1) bzw. 19,7\% (95-\%-KI: 16,9; 22,8). Die Rate war durchgängig in allen Altersgruppen und für alle Schweregrade der Einsamkeit höher als in der Allgemeinbevölkerung. Jüngeres Lebensalter, vollständige Erblindung, Vorliegen weiterer Beeinträchtigungen, Arbeitslosigkeit sowie Mobbing- und Gewalterfahrungen waren mit Einsamkeit assoziiert. Zugleich waren höhere Einsamkeits-Scores mit geringerer Lebenszufriedenheit assoziiert (vollständig angepasstes $\beta=-0,48 ; 95-\%-K l:-0,55 ;-0,41$ ).

Schlussfolgerungen: Einsamkeit ist bei Erwachsenen mit SB weit verbreitet. Strategien, die geeignet sind, die Einsamkeit zu lindern, könnten die Lebenszufriedenheit der Menschen, die mit SB oder Blindheit leben, verbessern.

(c) The Author(s). 2019

\section{Einleitung}

Eine Sehbehinderung (SB) ist eine erhebliche und oft irreversible Beeinträchtigung der Sehschärfe oder des Gesichtsfeldes [1]. Man unterscheidet grundsätzlich zwischen kongenitaler und erworbener SB sowie zwischen mittelschwerer SB, schwerer SB und Blindheit $[1,2]$. Einsamkeit kann für Menschen mit SB ein besonderes
Problem darstellen, da das Sehen ein sehr wichtiger Sinn für die zwischenmenschliche Interaktion und soziale Kommunikation ist. Menschen, die sehbehindert bzw. blind sind, haben weniger Möglichkeiten, soziale Fähigkeiten zu erlernen und zu verändern [3]. Darüber hinaus unterliegen Menschen mit SB einem erhöhten Risiko für Invalidität $[4,5]$, schlechten Gesundheitszustand [5, 6], Arbeitslosigkeit [4,5], niedriges Einkommen [5] und unerwünsch-

\section{KARGER}

Fax +4976145207 14

information@karger.com

www.karger.com

\section{(c) 2019 S. Karger GmbH, Freiburg}

Accessible online at:

www.karger.com/kop
Audun Brunes

Section for Trauma, Catastrophes and Forced Migration - Adults and Elderly

Norwegian Centre for Violence and Traumatic Stress Studies

PB 181 Nydalen, Oslo, Norwegen

audun.brunes@nkvts.no 
te zwischenmenschliche Ereignisse [7, 8] - alles Faktoren, die stark mit Einsamkeit korrelieren $[9,10]$.

Die Prävalenz von und die Risikofaktoren für Einsamkeit bei Menschen mit SB sind in mehreren Studien untersucht worden [11-18]. In den meisten davon bestanden die Populationen allerdings aus älteren Menschen. Zu Menschen mit SB im jungen und mittleren Erwachsenenalter liegen nur wenige Untersuchungen vor. Zugleich gibt die vorhandene Literatur keinen Aufschluss über soziodemographische und SB-assoziierte Merkmale als Risikofaktoren für Einsamkeit [11-13, 16], und in keiner der Studien wurden Faktoren wie z.B. das Durchleben traumatischer Ereignisse berücksichtigt. Ein besseres Verständnis der Prädiktoren für Einsamkeit bei sehbehinderten und blinden Menschen ist wichtig und potenziell hilfreich, um professionelle Hilfe gezielt denjenigen anzubieten, die sie benötigen.

Einsamkeit ist ein starker Prädiktor für die Gesundheit und Lebensqualität [10, 19-21]. Untersuchungen an der erwachsenen Allgemeinbevölkerung haben ergeben, dass bei einsamen Menschen die Lebenszufriedenheit insgesamt geringer war als bei nicht einsamen Menschen [21]. Unseres Wissens wurde jedoch bisher in keiner Studie der mögliche Zusammenhang zwischen Einsamkeit und Lebensqualität in Populationen mit SB untersucht.

Um diese Wissenslücke zu schließen, führten wir eine Querschnittsstudie mit einer Wahrscheinlichkeitsstichprobe von Erwachsenen mit SB durch. Mit der hier vorgestellten Studie wurden 3 grundlegende Ziele verfolgt: die Prävalenz von Einsamkeit bei Erwachsenen mit SB zu ermitteln, den Zusammenhang zwischen Einsamkeit und soziodemographischen Faktoren, Merkmalen der SB und Erfahrungen mit Mobbing und körperlicher bzw. sexualisierter Misshandlung sowie den Zusammenhang zwischen Einsamkeit und Lebenszufriedenheit zu beschreiben.

\section{Methoden}

\section{Aufbau und Teilnehmer Population mit SB}

In diese Querschnittsstudie wurden erwachsene ( $\geq 18$ Jahre) Mitglieder des Norwegischen Verbandes der Sehbehinderten und Blinden eingeschlossen. Um Vollmitglied dieses Verbandes zu werden, muss man eine medizinische Dokumentation einer SB bzw. einer irreversiblen, zu SB führenden Augenerkrankung vorweisen [22]. Von der Studie ausgeschlossen wurden Personen, die taub waren, schwere Sprachstörungen hatten und/oder weder eine skandinavische Sprache noch Englisch sprachen. Die Datenerhebung erfolgte in strukturierten Telefoninterviews zwischen Januar und Mai 2017. Alle Gespräche wurden von geschulten Interviewern eines Umfrageunternehmens durchgeführt. Die Mehrheit der Verbandsmitglieder war mittleren oder höheren Alters [22]. Um sicherzustellen, dass auch die jüngsten Patienten gleichberechtigt vertreten waren, wurden die Studienteilnehmer in 4 Altersgruppen eingeteilt (18-35, 36-50, 51-65, $\geq 66$ Jahre), und aus jeder Gruppe wurden etwa gleich viele Mitglieder nach dem Zufallsprinzip ausgewählt. Von den 1216 Personen, die kontaktiert wurden, nahmen 736 (61\%) teil und beantworteten die
Interviewfragen. Ein Flussdiagramm der Stichprobenauswahl wurde an anderer Stelle veröffentlicht [8].

\section{Allgemeinbevölkerung}

Wir extrahierten Normdaten über Einsamkeit und verschiedene soziodemographische Faktoren aus der LOGG-Studie (Life Course Gender and Generation) [23]. Die Teilnehmer der LOGGStudie waren eine national repräsentative Stichprobe der nicht institutionalisierten Erwachsenen im Alter von 18-79 Jahren in Norwegen. Die Datenerhebung erfolgte zwischen Januar 2007 und Januar 2009 im Rahmen von Telefoninterviews, selbstausgefüllten Brieffragebögen sowie nationalen Registern. Von den 25368 Personen, die die Teilnahmevoraussetzungen erfüllten, absolvierten $14884(60,0 \%)$ das Telefoninterview, und 10791 $(42,6 \%)$ beantworteten den Brieffragebogen und sandten ihn ein. Wir hatten Zugriff auf alle Daten der LOGG-Studie. Der Datenzugriff wurde durch das Norwegische Zentrum für Forschungsdaten erteilt (Zugriffsnummer \#4392). Die LOGG-Studie war vom Regionalkomitee für Ethik in der medizinischen und Gesundheitsforschung genehmigt. Von allen Teilnehmern wurde eine Einverständniserklärung nach Aufklärung eingeholt.

\section{Messgrößen}

Einsamkeit

Das Ausmaß der Einsamkeit wurde bei der SB-Population mit dem TILS-Instrument (Three-Item Loneliness Scale) gemessen. Diese Skala wurde für Populationsbefragungen entwickelt und zeigt gute interne Konsistenz $(r=0,72)$ und hohe konkurrente Validität [24]. Die Skala beruht auf den folgenden 3 Fragen: «Wie oft fehlt Ihnen ein Mensch an Ihrer Seite?», «Wie oft fühlen Sie sich sozial ausgegrenzt?», «Wie oft fühlen Sie sich sozial isoliert?». Die Antwortkategorien wurden mit 1 («fast nie»), 2 («manchmal») und 3 («oft») codiert. Der Chronbachs-Alpha-Wert der Skala betrug 0,81. Durch Aufaddierung der 3 Items wurde ein Summenscore ermittelt (Wertebereich 3-9). Zusätzlich zu diesem kontinuierlichen Einsamkeits-Score legten wir auch eine kategorische Einstufung fest. Ein Summenscore von 5 oder 6 wurde als «mittelschwere Einsamkeit» eingestuft und ein Score $\geq 7$ als «schwere Einsamkeit» [25].

Die verwendeten Parameter zur Beurteilung und Klassifizierung der Einsamkeit in der Allgemeinbevölkerung sind in Suppl. Tab. 1 (www.karger.com/?DOI=502498) beschrieben.

\section{Lebenszufriedenheit}

"Cantril's Ladder of Life Satisfaction» wurde im Fragebogen verwendet, um die aktuelle Lebenszufriedenheit in der SB-Population zu messen [26]. Die Teilnehmer wurden gebeten, sich eine Leiter mit 10 Sprossen vorzustellen, wobei die unterste Sprosse für das schlechtestmögliche Leben steht (Punktzahl = 1) und die oberste für das bestmögliche (Punktzahl =10).

\section{Unabhängige Variablen}

Sowohl die SB-Population als auch die Allgemeinbevölkerung wurden hinsichtlich verschiedener soziodemographischer Merk- 
Tab. 1. Merkmale der Studienpopulation mit Sehbehinderung $(N=736)$ und der untersuchten Allgemeinbevölkerung $(N=14884)$

\begin{tabular}{|c|c|c|c|}
\hline \multirow[b]{2}{*}{ Merkmal } & \multirow{2}{*}{$\frac{\text { SB-Population }}{n(\%)}$} & \multicolumn{2}{|c|}{ Allgemeinbevölkerung } \\
\hline & & n (\%) & $x^{2} ; p$-Wert ${ }^{\#}$ \\
\hline \multicolumn{3}{|l|}{ Alter (Jahre) } & 86,$5 ; p<0,001$ \\
\hline $18-35$ & $157(21,3)$ & $4265(28,7)$ & \\
\hline $36-50$ & $186(25,3)$ & $4568(30,7)$ & \\
\hline $51-65$ & $200(27,2)$ & $3914(26,3)$ & \\
\hline$\geq 66$ & $193(26,2)$ & $2137(14,4)$ & \\
\hline \multicolumn{3}{|l|}{ Geschlecht } & 8,$3 ; p=0,004$ \\
\hline Frauen & $333(45,2)$ & $7545(50,7)$ & \\
\hline Männer & $403(54,8)$ & $7339(49,3)$ & \\
\hline \multicolumn{4}{|l|}{ Ausbildung (Jahre) } \\
\hline$<11$ & $115(15,6)$ & $2820(19,0)$ & \\
\hline $11-13$ & $286(38,9)$ & $6849(46,0)$ & \\
\hline$\geq 14$ & $335(45,5)$ & $5215(35,0)$ & \\
\hline \multicolumn{3}{|l|}{ Familienstand } & 115,$8 ; p=0,001$ \\
\hline Verheiratet/Partnerschaft & $347(47,2)$ & $9580(64,4)$ & \\
\hline Alleinstehend & $260(35,3)$ & $2953(19,8)$ & \\
\hline Geschieden/verwitwet & $129(17,5)$ & $2351(15,8)$ & \\
\hline \multicolumn{3}{|l|}{ Berufsstatus } & 522,$1 ; p=0,001$ \\
\hline Angestellt & $295(40,1)$ & $11061(74,7)$ & \\
\hline Arbeitslos & $271(36,8)$ & $1633(7,8)$ & \\
\hline Im Ruhestand & $170(23,1)$ & $2622(17,7)$ & \\
\hline
\end{tabular}

male ausgewertet, darunter Alter (18-35, 36-50, 51-65, $\geq 66$ Jahre), Geschlecht, Bildungsstand ( $<11,11-13, \geq 14$ Jahre), Familienstand (ledig, verheiratet/Partnerschaft, geschieden/verwitwet) und Berufsstand (berufstätig/in Ausbildung, arbeitslos, im Ruhestand).

Die SB-Population wurde außerdem danach befragt, wie viele Personen in ihrem Haushalt lebten $(1, \geq 2)$, ob sie schon einmal Mobbing oder körperliche bzw. sexualisierte Gewalt erlebt haben (keine, nur Mobbing, Mobbing und Gewalt), ob sie weitere Beeinträchtigungen haben (nein, ja), wie ausgeprägt ihr Sehverlust ist (mittelschwer/nicht näher bestimmt, schwer, Blindheit) und wie der aktuelle Status ihrer SB ist (stabil, progressiv). Als weitere Variable leiteten wir das «Alter zu Beginn der $\mathrm{SB}$ » her, indem wir vom Alter des Teilnehmers die Zahl der Jahre, die er insgesamt schon mit einer SB lebte, abzogen. Diese Variable wurde in die folgenden 3 Kategorien eingeteilt: «angeboren» (0 Jahre), «Kindheit/Jugend» (1-24 Jahre) und «Erwachsenenalter» ( $\geq 25$ Jahre).

\section{Statistische Methoden}

Für die deskriptive Statistik wurden Mittelwerte, Standardabweichungen (SD), Häufigkeiten und prozentuale Anteile verwendet. Differenzen bei Häufigkeitsangaben wurden mittels Pearson's Chi-Quadrat-Test beurteilt. Die Anteile der Personen mit mittelschwerer und schwerer Einsamkeit wurden pro Altersgruppe tabellarisch dargestellt. Alle Anteilsangaben sind Schätzer mit 95-\%-Konfidenzintervall (KI).

Generalisierte lineare Modelle (GLM) mit Gauß-Verteilung und einem Log-Link wurden verwendet, um unabhängige Zusam- menhänge zwischen soziodemographischen Faktoren, SB-Merkmalen und Erfahrungen mit Mobbing und körperlicher/sexualisierter Gewalt einerseits und Einsamkeits-Outcomes andererseits abzuschätzen. Bei diesen Analysen betrachteten wir Einsamkeit als nicht transformierte, kontinuierliche Variable. Die Anpassungsgüte des Modells beurteilten wir anhand des Akaike-Informationskriteriums und anhand von Residuendiagrammen [27]. Außerdem verwendeten wir Gauß-GLM, um den Zusammenhang zwischen Einsamkeit und Lebenszufriedenheit zu untersuchen [27]. Diese Modelle waren entweder nicht angepasst oder nach Alter, Geschlecht, Bildungs-, Berufs- und Familienstand, Anzahl der Personen im Haushalt, Mobbing- und körperlicher/ sexualisierter Gewalterfahrung, weiteren Beeinträchtigungen, Schwere der SB und Jahren seit Beginn der SB angepasst. Die Lebenszufriedenheit betrachteten wir in den Analysen als nicht transformierte, kontinuierliche Variable. Wir testeten auf eine lineare Dosis-Wirkungs-Beziehung, indem wir die log-Wahrscheinlichkeit im Modell mit Einsamkeit als kontinuierliche Variable mit der im Modell, in dem Einsamkeit in Quartile kategorisiert als kategorische Variable behandelt wurde, verglichen. Ein $p$-Wert $<0,05$ zeigte eine Abweichung von der Linearität an. Des Weiteren testeten wir auf Effektmaß-Modifikation zwischen Einsamkeit und den einzelnen Kovariablen und verwendeten dazu den Likelihood-Quotienten-Test zum Vergleich der Modelle mit und ohne den Produktterm [28].

Das Signifikanzniveau wurde auf $p=0,05$ festgelegt. Die statistischen Analysen wurden mit Stata Version 15 (Stata Corp., College Station, TX, USA) durchgeführt. 
Tab. 2. Prävalenz von Einsamkeit in der Population mit Sehbehinderung und in der Allgemeinbevölkerung

\begin{tabular}{|c|c|c|c|c|c|c|}
\hline \multirow[t]{2}{*}{ Altersklassen } & \multicolumn{2}{|c|}{$\begin{array}{l}\text { SB-Population }(n=736)^{\mathrm{a}} \\
\text { TILS }\end{array}$} & \multicolumn{2}{|c|}{ Einzelfrage } & \multicolumn{2}{|c|}{ DCG } \\
\hline & $\%$ & $95-\%-K I$ & $\%$ & $95-\%-K I$ & $\%$ & $95-\%-K I$ \\
\hline \multicolumn{7}{|c|}{ Mittelschwere Einsamkeit } \\
\hline 18-35 Jahre & 31,2 & $24,1-39,1$ & 19,6 & $18,4-20,8$ & 16,5 & $15,3-17,6$ \\
\hline 36-50 Jahre & 30,1 & $23,6-37,2$ & 15,8 & $14,8-16,9$ & 16,1 & $15,1-17,2$ \\
\hline 51-65 Jahre & 23,0 & $17,4-29,5$ & 17,3 & $16,1-18,5$ & 18,5 & $17,3-19,7$ \\
\hline$\geq 66$ Jahre & 31,1 & $24,6-38,1$ & 22,4 & $20,6-24,2$ & 23,3 & $21,5-25,1$ \\
\hline Gesamtpopulation & 28,7 & $25,4-32,1$ & 18,2 & $17,6-18,9$ & 17,9 & $17,2-18,5$ \\
\hline \multicolumn{7}{|l|}{ Schwere Einsamkeit } \\
\hline 18-35 Jahre & 21,0 & $14,9-28,2$ & 2,1 & $1,7-2,6$ & 4,9 & $4,3-5,6$ \\
\hline 36-50 Jahre & 27,9 & $20,7-33,9$ & 2,2 & $1,8-2,7$ & 6,1 & $5,5-6,9$ \\
\hline 51-65 Jahre & 19,0 & $13,8-25,1$ & 3,2 & $2,7-3,8$ & 6,0 & $5,3-6,8$ \\
\hline$\geq 66$ Jahre & 12,4 & $8,1-17,9$ & 3,8 & $3,0-4,7$ & 7,2 & $6,1-8,4$ \\
\hline Gesamtpopulation & 19,7 & $16,9-22,8$ & 2,7 & $2,4-3,0$ & 5,9 & $5,5-6,3$ \\
\hline
\end{tabular}

SB = Sehbehinderung, KI = Konfidenzintervall, TILS = Three Item Loneliness Scale, DCG = De Jong Gierveld Loneliness Scale. a Die Einzelfrage wurde 26-mal nicht beantwortet, der DCG-Fragebogen 122-mal.

\section{Ergebnisse}

43\% der SB-Population hatten eine angeborene SB, 57\% eine in der Kindheit oder im Erwachsenenalter erworbene. Bei rund einem Drittel der Teilnehmer lagen zusätzliche Beeinträchtigungen vor. Das Alter bei Auftreten der SB lag zwischen 0 und 76 Jahren (Mittelwert: 19 Jahre). 35\% hatten eine mittelschwere/sonstige SB, $40 \%$ eine schwere, und $25 \%$ waren blind. Tabelle 1 zeigt die Merkmale der SB-Studienpopulation und der untersuchten Allgemeinbevölkerung. Die beiden Populationen wiesen signifikante Unterschiede bei allen 5 Studienmerkmalen auf $(p<0,05)$.

Der mittlere Einsamkeits-Score betrug 4,83 (SD 1,82) - 4,88 bei den Frauen und 4,78 bei den Männern $(p=0,46)$. Die Mehrheit der Teilnehmer gab an, dass ihnen manchmal (39,0\%) oder oft $(21,1 \%)$ ein Mensch an ihrer Seite fehlte. Darüber hinaus antworteten viele Teilnehmer, dass sie sich manchmal $(29,2 \%)$ oder oft $(12,2 \%)$ sozial ausgegrenzt und manchmal $(27,0 \%)$ oder oft $(13,3 \%)$ sozial isoliert fühlten. Wie auch Tabelle 2 zeigt, war der Anteil der Personen, die als einsam eingestuft wurden, in der SB-Population höher als in der Allgemeinbevölkerung. Dies galt durchgängig für alle Altersgruppen und alle Schweregrade der Einsamkeit.

Tabelle 3 zeigt die Ergebnisse der nicht angepassten und angepassten Regressionsanalysen für Einsamkeit und die verschiedenen soziodemographischen Faktoren, SB-Merkmale und Erfahrungen mit Mobbing und körperlicher und sexualisierter Gewalt. In den nicht angepassten Analysen waren alle Faktoren außer Geschlecht und SB-Stabilität signifikant mit Einsamkeit assoziiert. In den angepassten Modellen waren Mobbing-Erfahrung und die Altersgruppe der 36- bis 50-Jährigen am stärksten mit Einsamkeit assoziiert. Hohe mittlere Einsamkeits-Scores lagen außerdem bei den Personen vor, die vollständig blind waren, zusätzliche Beeinträchtigungen aufwiesen, körperliche oder sexualisierte Gewalt erlebt hatten, arbeitslos, geschieden oder verwitwet waren oder 11-13 Jahre Schul-/Ausbildung hatten.
Höhere Einsamkeits-Scores waren mit geringerer Lebenszufriedenheit assoziiert ( $\beta=-0,48$; 95-\%-KI: $-0,55 ;-0,41)$. Diese Assoziation war nicht-linear $(p=0,002)$, wobei im oberen Bereich der Einsamkeitsskala Veränderungen beim Einsamkeits-Score mit einer größeren Veränderung bei der Lebenszufriedenheit einhergingen (Suppl. Abb. 1). Nach den Anpassungen blieben die Schätzwerte unverändert (vollständig angepasstes $\beta=-0,44$; 95-\%-KI: -0,52; -0,36). Darüber hinaus stellten wir einen signifikanten Produktterm zwischen Einsamkeit und Geschlecht fest $\left(\chi^{2}=4,2 ; p=0,04\right)$, woraus sich bei den Frauen eine geringfügig stärkere Assoziation ergab (vollständig angepasstes $\beta=-0,56$; 95-\%-KI: $-0,66 ;-0,45)$ als bei den Männern (vollständig angepasstes $\beta=-0,36$; $95-\%-\mathrm{KI}:-0,45 ;-0,21)$. Keine weiteren Produktterme erreichten statistische Signifikanz $(p>0,05)$.

\section{Diskussion}

Unsere Ergebnisse belegen, dass fast jeder zweite Erwachsene mit $\mathrm{SB}$ von mittelschwerer oder schwerer Einsamkeit betroffen ist und der Anteil in allen Altersgruppen durchgängig höher ist als in der Allgemeinbevölkerung. Zusätzlich erhöht war das Einsamkeitsrisiko in der Altersgruppe von 36 bis 50 Jahren sowie bei denen, die Mobbing, körperliche oder sexualisierte Gewalt erlebt hatten, blind waren, weitere Beeinträchtigungen aufwiesen oder arbeitslos waren. Schließlich war ein hohes Maß an Einsamkeit mit einer geringeren Lebenszufriedenheit assoziiert.

\section{Interpretation und Vergleich}

Die hohen Einsamkeitsraten in unserer Studie decken sich mit Studienergebnissen zu Menschen mit SB in den Niederlanden [11, 12], Island [16] und Finnland [14] und sind zwei- bis dreimal höher als in Studien aus den USA [13] und Kanada [18]. Darüber hinaus untermauern die im Vergleich zur Allgemeinbevölkerung durch- 
Tab. 3. Regressionsanalysen zur Assoziation von Einsamkeit mit verschiedenen Faktoren in der Population mit Sehbehinderung $(n=736)$

\begin{tabular}{|c|c|c|}
\hline Unabhängige Variablen & $\begin{array}{l}\text { Nicht angepasst }{ }^{\mathrm{a}} \\
\text { Beta }(95-\%-\mathrm{KI})\end{array}$ & $\begin{array}{l}\text { Angepasst }{ }^{\mathrm{a}} \\
\text { Beta }(95-\%-K I)\end{array}$ \\
\hline \multicolumn{3}{|l|}{ Alter (Jahre) } \\
\hline $18-35$ vs. $\geq 66$ & $-0,44(0,05 ; 0,83)$ & $0,41(-0,28 ; 1,11)$ \\
\hline $36-50$ vs. $\geq 66$ & $-0,74(0,37 ; 1,11)$ & $0,77(0,12 ; 1,43)$ \\
\hline $51-65$ vs. $\geq 66$ & $0,20(-0,16 ; 0,56)$ & $0,23(-0,40 ; 0,86)$ \\
\hline Geschlecht: weiblich vs. männlich & $0,10(-0,17 ; 0,37)$ & $-0,10(-0,36 ; 0,15)$ \\
\hline \multicolumn{3}{|l|}{ Bildung (Jahre) } \\
\hline$<11$ Vs. $\geq 14$ & $0,32(-0,07 ; 0,71)$ & $0,16(-0,21 ; 0,53)$ \\
\hline $11-13$ vs. $\geq 14$ & $0,59(0,30 ; 0,88)$ & $0,35(0,07 ; 0,62)$ \\
\hline Familienstand: Alleinstehend vs. verheiratet/Partner & $0,89(0,60 ; 1,17)$ & $0,60(0,16 ; 1,03)$ \\
\hline Geschieden/verwitwet vs. verheiratet/Partner & $1,01(0,64 ; 1,37)$ & $1,12(0,64 ; 1,59)$ \\
\hline Haushaltsmitglieder: 1 vs. $\geq 2$ & $0,66(0,39 ; 0,93)$ & $-0,10(-0,51 ; 0,31)$ \\
\hline Berufsstatus: Arbeitslos vs. berufstätig/in Ausbildung & $0,80(0,50 ; 1,10)$ & $0,50(0,20 ; 0,81)$ \\
\hline Ruhestand vs. berufstätig/in Ausbildung & $-0,07(-0,41 ; 0,28)$ & $0,36(-0,30 ; 1,02)$ \\
\hline Sonstige Beeinträchtigungen: ja vs. nein & $0,91(0,64 ; 1,19)$ & $0,66(0,39 ; 0,92)$ \\
\hline Schweregrad der SB: Schwere vs. mittelschwere SB & $0,28(-0,03 ; 0,59)$ & $0,16(-0,13 ; 0,46)$ \\
\hline Blindheit vs. mittelschwere SB & $0,37(0,02 ; 0,78)$ & $0,39(0,05 ; 0,73)$ \\
\hline Alter bei Auftreten der SB: angeboren vs. Erwachsenenalter & $0,30(-0,00 ; 0,60)$ & $0,09(-0,22 ; 0,41)$ \\
\hline Kindheit/Jugend vs. Erwachsenenalter & $0,34(-0,03 ; 0,72)$ & $0,11(-0,27 ; 0,48)$ \\
\hline SB-Stabilität: progressiv vs. stabil & $0,13(-0,17 ; 0,43)$ & $0,15(-0,14 ; 0,43)$ \\
\hline Mobbing- oder Gewalterfahrung: Mobbing vs. keine & $0,92(0,62 ; 1,22)$ & $0,76(0,46 ; 1,06)$ \\
\hline Gewalt vs. keine & $1,18(0,85 ; 1,52)$ & $0,77(0,43 ; 1,10)$ \\
\hline
\end{tabular}

gängig höheren Einsamkeitsraten in allen Altersgruppen die Beobachtung, dass Menschen mit SB einem höheren Einsamkeitsrisiko unterliegen als Menschen mit unbeeinträchtigter Sehfähigkeit $[11,14,17,18]$.

Unsere Ergebnisse stimmen zudem mit denen von Karlsson [16] überein, die belegen, dass blinde Menschen einsamer sind als Menschen mit mittelschwerer bis schwerer SB. Ähnlich wie in dieser Studie wurde auch in unserer Studie bei der Mehrheit der Teilnehmer die SB in der Kindheit oder dem frühen Erwachsenenalter diagnostiziert. Studien mit Teilnehmern, die ihre Sehkraft später im Leben verloren hatten, erbrachten gemischte Ergebnisse im Hinblick darauf, ob die Schwere der SB mit der der Einsamkeit assoziiert ist [11-13].

Dass die Einsamkeit bei den 36- bis 50-Jährigen am ausgeprägtesten ist, ist ein Unterschied zur Allgemeinbevölkerung, wo Jugendliche und ältere Menschen am stärksten von Einsamkeit betroffen sind [29]. Unsere Ergebnisse lassen sich im Sinne der allgemein hohen Erfolgserwartungen interpretieren, die in dieser Lebensphase im Familienleben, in sozialen Kontexten und im Berufsleben herrschen. Das Gefühl der Einsamkeit resultiert aus kognitiven Beurteilungen, bei denen die betroffene Person die tatsächlichen Gegebenheiten mit ihren Erwartungen und Bedürfnissen abgleicht [2]. Im hohen Lebensalter sind die Anforderungen an das Sehvermögen möglicherweise nicht mehr von so entscheidender Bedeutung. Darüber hinaus hatten mehrere Teilnehmer ihr Sehvermögen in höherem Alter eingebüßt, und ein Teil von ihnen hatte möglicherweise im Laufe ihres langen Lebens bereits soziale Netzwerke aufgebaut. Diese Hypothesen konnten wir jedoch nicht empirisch prüfen.

Das höhere Maß an Einsamkeit bei arbeitslosen Personen stützt die Ergebnisse einer früheren Untersuchung [15], in der 50\% der Personen mit SB der Aussage zustimmten, dass die Arbeitslosigkeit ihr soziales Netzwerk reduzierte und zum Gefühl sozialer Ausgrenzung führte. Mangelnde unterstützende Rahmenbedingungen hielten die Menschen mit SB davon ab, am gesellschaftlichen Leben teilzunehmen [30]; dieser Effekt könnte durch die persönlichen, sozialen und finanziellen Folgen der Erwerbslosigkeit noch verstärkt werden [31].

Ebenso wie in der Allgemeinbevölkerung [32] stellten wir fest, dass das Erleben von Mobbing oder Gewalt eng mit Gefühlen von Einsamkeit assoziiert ist. Solche negativen zwischenmenschlichen Erfahrungen können Gefühle sozialer Entfremdung, persistierende negative Gedanken und Emotionen, verzerrte Schuldzuweisungen an sich selbst oder andere sowie Vertrauensverlust in sich selbst, in andere oder in die Welt im Allgemeinen hervorrufen [33]. So können Schwierigkeiten bei der Bewältigung von Stresssituationen zu Vermeidungsverhalten und Rückzug aus sozialen Situationen führen, was wiederum in dauerhaften Einsamkeitsgefühlen resultieren kann [29].

Die starke Assoziation zwischen Einsamkeit und verminderter Lebenszufriedenheit ist ebenso in der Allgemeinbevölkerung dokumentiert [21], was einmal mehr unterstreicht, wie wichtig das Sozialleben und das Gefühl des Eingebundenseins für die Lebensqualität sind. Soziale Interaktion gilt als integraler Bestand- 
teil eines erfüllten Lebens und unerfüllte Bedürfnisse können dazu führen, dass das Leben als weniger schön und sinnerfüllt angesehen wird [34]. Die Kausalität kann aber auch umgekehrt sein, insofern dass Menschen, die mit ihrem Leben weniger zufrieden sind, möglicherweise stärker zum sozialen Rückzug neigen [21].

\section{Stärken und Einschränkungen}

Stärken dieser Studie waren ihr großer Stichprobenumfang, die Technik der Wahrscheinlichkeitsstichprobe, der Einsatz interviewbasierter Methoden mit validierten Instrumenten sowie die detaillierten Informationen zu mehreren SB-bezogenen Merkmalen. Durch die überdurchschnittliche Erfassung jüngerer Erwachsener konnten wir valide Schätzer für Einsamkeit über ein breites Spektrum von Altersgruppen hinweg ermitteln.

Nicht adressieren konnten wir in dieser Querschnittsstudie Kausalzusammenhänge, und obwohl wir für mehrere potenzielle Störfaktoren Kontrollen angewendet haben, ist dennoch möglich, dass unsere Analysen von residualer Konfundierung betroffen sind. Darüber hinaus können die subjektiven Angaben der Betroffenen zu ihrer SB und anderen wichtigen Faktoren die Genauigkeit und Gültigkeit der Schätzungen beeinträchtigt haben. Das TILS-Instrument gilt zwar als zuverlässig zur Beurteilung von Einsamkeit [35], jedoch fehlt ein standardisierter Cut-OffWert, ab dem Personen als «einsam» eingestuft werden. Dass wir einen eher konservativen Wert für schwere Einsamkeit verwendet haben, könnte zu den niedrigen Schätzern in dieser speziellen Kategorie geführt haben [25]. Darüber hinaus könnte partielle Nicht-Teilnahme zu Verzerrungen bei den Schätzern geführt haben. Verzerrungen bei der Probenauswahl könnten insbesondere die Häufigkeit von Einsamkeit und Kovariablen sowie in geringerem Maße auch den untersuchten Zusammenhang beeinflusst haben [36]. Und nicht zuletzt wirft die Rekrutierung der Teilnehmer aus einem Blinden- und Sehbehindertenverband die Frage nach der Repräsentativität unserer Studienstichprobe auf. Bezogen auf die Volkszählungsdaten der norwegischen Statistikbehörde von 2015 [37] wies unsere Stichprobe keine Abweichungen in Bezug auf Geschlecht, Berufsstatus und geographischen Standort auf. Unsere Stichprobe enthielt jedoch einen erhöhten prozentualen Anteil von Menschen, die blind, hochgebildet und allein lebend waren.

\section{Bedeutung}

Unsere Ergebnisse deuten darauf hin, dass die Bewältigung des Soziallebens erschwert sein kann, wenn das Sehvermögen beeinträchtigt ist, und dass Menschen mit SB potenziell eher ausgegrenzt und isoliert werden. Hürden auszuräumen, die die soziale Teilhabe und Integration erschweren, sollte ein prioritäres Ziel sein, um soziale Ausgrenzung und Einsamkeit zu verhindern. Solche Hürden können sowohl in allgemeinen Einstellungen liegen als auch in Rechtsvorschriften und sozialen, kulturellen und physischen Strukturen. Hier kommen die Konzepte der Barrierefreiheit und des Universellen Designs ins Spiel [30]. Darüber hinaus untermauern die potenziellen Auswirkungen von Mobbing und Missbrauch auf den Faktor Einsamkeit die Notwendigkeit sowohl von Präventivmaßnahmen als auch von professioneller Begleitung nach entsprechenden Erlebnissen.

Mit dem Begriff «Information Deprivation Trauma» wird in der wissenschaftlichen Literatur zu schwerhörigen und gehörlosen Menschen beschrieben, wie ein Mangel an Informationen es erschwert, in einer gegebenen Situation angemessen zu handeln [38]. Das Leben wird dadurch weniger vorhersehbar und kontrollierbar. Unter einem solchen Informationsmangel leiden mit hoher Wahrscheinlichkeit auch Menschen mit SB und erfahren dadurch vermehrt Entfremdung, Ausgrenzung und Einsamkeit kausale Beziehungen, die sich potenziell gegenseitig verstärken. Darum sind Maßnahmen erforderlich, um den Zugang zu wichtigen Lebensbereichen zu verbessern, von Gebäuden über Straßen und Verkehr bis hin zur Kommunikation [30]. Von besonderem Interesse für Ärzte ist hierbei, dass die begrenzte soziale Vernetzung in Kombination mit allgemeinem Informationsmangel auch bedeuten kann, dass Menschen mit SB vermehrt Probleme beim Zugang zur Gesundheitsversorgung haben und mit erhöhter Wahrscheinlichkeit suboptimal medizinisch behandelt werden. Medizinische Fachkräfte sollten sich daher der besonderen $\mathrm{He}$ rausforderungen für Menschen mit SB und der Bedeutung guter Kommunikation und Aufklärung bewusst sein.

\section{Schlussfolgerungen}

Diese Querschnittsstudie liefert neue Erkenntnisse über Einsamkeit bei Menschen mit SB. Menschen mit SB unterliegen in allen Altersgruppen einem erhöhten Risiko für Einsamkeit. Wir haben festgestellt, dass die Einsamkeit stark mit niedrigem Alter, Schwere der Beeinträchtigung, Arbeitslosigkeit sowie Mobbing- und Gewalterfahrungen assoziiert ist. Insgesamt ging Einsamkeit mit einer geringeren Lebenszufriedenheit einher. Strategien zur Förderung der sozialen Teilhabe und des Zugangs für Menschen mit SB könnten für die Steigerung des allgemeinen Wohlbefindens dieser Population von Nutzen sein.

\section{Online Supplemental Material}

Suppl. Tab. 1. Klassifizierung von Einsamkeit in den verschiedenen Messgrößen

Suppl. Abb. 1. Zusammenhang zwischen Einsamkeit und Lebenszufriedenheit: Running-Smoother und korrespondierende 95\%-Konfidenzintervalle des Zusammenhangs zwischen Einsamkeit und Lebenszufriedenheit in der Population der Menschen mit Sehbehinderung $(\mathrm{n}=736)$.

Suppl. Tab. 2. Im Interview einbezogene Themengebiete: Erfasste Daten aus dem strukturierten Interview

Das Supplemental Material finden Sie unter www.karger.com/?DOI=502498.

\section{Danksagung}

Die Autoren/-innen danken allen kooperierenden Projektpartnern im European Network for Psychosocial Crisis Management - Assisting Disabled in Case of Disaster (EUNAD), die die Durchführung dieser Umfrage mög- 
lich gemacht haben. Wir danken außerdem dem Norwegischen Zentrum für Forschungsdaten für den Zugang zu den LOGG-Studiendaten. Die LOGGStudie wurde finanziert durch den Norwegischen Forschungsrat (Förderprojekt Nr. 168373), die Ministerien für Gesundheit und Pflege, für Arbeit, für Jugend, Chancengleichheit und Inklusion sowie für Lokalpolitik und regionale Entwicklung, das norwegische sozialwissenschaftliche Forschungsinstitut NOVA und die norwegische Statistikbehörde. Der LOGGDatensatz ist Teil des Projekts ACCESS Life Course Infrastructure, das durch den Nationalen Finanzierungsinitiative für die Forschungsinfrastruktur des Norwegischen Forschungsrates (Förderprojekt Nr. 195403) und durch NOVA finanziert wurde. Keine der vorgenannten Institutionen ist für die in diesem Artikel dargestellten Datenanalyse oder Interpretationen verantwortlich.

\section{Finanzierung}

Diese Arbeit wurde von der Europäischen Kommission, Generaldirektion Katastrophenschutz und humanitäre Hilfe (Förderprojekt Nr. ECHO/ SUB/2015/718665/PREP17) und dem norwegischen Verband der Sehbehinderten und Blinden finanziert.

\section{Verfügbarkeit von Daten und Materialien}

Die Daten stammen aus dem Forschungsprojekt European Network for Psychosocial Crisis Management - Assisting Disabled in Case of Disaster (EUNAD). Öffentliche Verfügbarkeit könnte die Privatsphäre der Befragten beeinträchtigen. Gemäß der von allen Befragten eingeholten Einwilligung nach Aufklärung sind die Daten ordnungsgemäß und in Übereinstimmung mit dem norwegischen Datenschutzgesetz zu speichern. Anonymisierte Daten hingegen werden Forschern zugänglich gemacht, die einen methodisch fundierten Antrag in Übereinstimmung mit der Einwilligung der Befragten vorlegen. Interessierte Forscher können beim Projektleiter Trond Heir (trond. heir@medisin.uio.no) unsere Studiendaten anfordern.

\section{Beiträge der einzelnen Autoren}

AB trug zur Datenanalyse, Interpretation, zum Verfassen des Artikels und zur Formatbearbeitung bei; MBH trug zur Konzeption und zum Design der Studie, zum Verfassen und zur finalen Freigabe des Artikels bei; TH trug zur Konzeption und zum Design der Studie, zur Datenanalyse und Interpretation, zum Verfassen und zur finalen Freigabe des Artikels bei.

\section{Genehmigung durch Ethikkommission und Einwilligung zur Teilnahme}

Die Studie wurde anonym durchgeführt; das Regionalkomitee für Ethik in der medizinischen und Gesundheitsforschung forderte auf Anfrage keine weitere formelle ethische Genehmigung (Referenznummer: 2016/1615A). Alle Teilnehmer erteilten nach Aufklärung ihre Einwilligung zur Teilnahme an der Studie. Die Teilnahme an der Studie war freiwillig und die Teilnehmer waren darüber informiert, dass sie jederzeit aus der Studie ausscheiden konnten.

\section{Disclosure Statement}

Die Autoren erklären, dass keine Interessenkonflikte bestehen.

\section{Lizenzangabe}

Audun Brunes, Marianne B. Hansen, Trond Heir: Loneliness among adults with visual impairment: prevalence, associated factors, and relationship to life satisfaction. Health Qual Life Outcomes 2019;17:24 (https://doi. org/10.1186/s12955-019-1096-y), ${ }^{\circ}$ The Author(s), 2019 (Übersetzung, «Additional file», «Abbrevations», «Consent for Publication» und «Publisher's Note» gekürt, Hinweis auf Online Supplementary Material eingefügt), lizensiert unter CC BY 4.0 (https://creativecommongs.org/licenses/by/4.0/deed.de).

\section{Literatur}

1 Colenbrander A: Assessment of functional vision and its rehabilitation. Acta Ophthalmol. 2010;88:163-173.

2 World Health Organization: International classification of diseases and health problems 10th revision. World Health Organization, 2016, http://apps.who.int/classifications/icd10/ browse/2016/en/ (Zugriff 25.05.2018).

3 Jindal-Snape D: Generalization and maintenance of social skills of children with visual impairments: self-evaluation and the role of feedback. JVIB 2004;98:1-23.

4 Fenwick E, Rees G, Pesudovs K, et al.: Social and emotional impact of diabetic retinopathy: a review. Clin Exp Ophthalmol 2012;40: 27-38.

5 Cumberland PM, Rahi JS: Visual function, social position, and health and life chances: the UK biobank study. JAMA Ophthalmol 2016; 134:959-966.

6 Crews JE, Chou C-F, Zack MM, et al.: The association of health-related quality of life with severity of visual impairment among people aged 40-64 years: findings from the 20062010 behavioral risk factor surveillance system. Ophthalmic Epidemiol 2016;23:145-153.

7 Brunes A, Hansen MB, Heir T: Post-traumatic stress reactions among individuals with visual impairments: a systematic review. Disabil Rehabil 2018:12:1-8.

$>8$ Brunes A, Nielsen MB, Heir T: Bullying among people with visual impairment: prevalence, associated factors and relationship to self-efficacy and life satisfaction. World J Psychiatry 2018;8:43.

$>9$ Cohen-Mansfield J, Hazan H, Lerman Y, et al.: Correlates and predictors of loneliness in older-adults: a review of quantitative results informed by qualitative insights. Int Psychogeriatr 2016;28:557-576.

10 Beutel ME, Klein EM, Brähler E, et al.: Loneliness in the general population: prevalence, determinants and relations to mental health. BMC Psychiatry 2017;17:97.

11 Alma MA, Van der Mei SF, Feitsma WN, et al.: Loneliness and self-management abilities in the visually impaired elderly. J Aging Health 2011;23:843-861.

12 Verstraten P, Brinkmann W, Stevens N, et al.: Loneliness, adaptation to vision impairment, social support and depression among visually impaired elderly. Int Congr Ser 2005;1282: 317-321.

13 Evans RL: Loneliness, depression, and social activity after determination of legal blindness. Psychol Rep 1983;52:603-608.

14 Savikko N, Routasalo P, Tilvis RS, et al.: Predictors and subjective causes of loneliness in an aged population. Arch Gerontol Geriatr 2005;41:223-233.

15 Andersen TK, Skarholt K: Med jobb i skikte: En studie av hva som påvirker synshemmedes yrkesdeltagelse og mulighetsrom i arbeidslivet. Trondheim, SINTEF, 2014, http://senterforlikestilling.org/sintef-rapport-med-jobb-i-sikte (Zugriff 07.06.2017).

16 Karlsson JS: Self-reports of psychological distress in connection with various degrees of visual impairment. JVIB 1998;92:483-490. 
17 Wahl HW, Heyl V, Drapaniotis PM, et al.: Severe vision and hearing impairment and successful aging: a multidimensional view. Gerontologist 2013;53:950-962.

18 Guthrie DM, Davidson JG, Williams N, et al.: Combined impairments in vision, hearing and cognition are associated with greater levels of functional and communication difficulties than cognitive impairment alone: analysis of interRAI data for home care and long-term care recipients in Ontario. PLoS One 2018; 13:e0192971.

19 Petitte T, Mallow J, Barnes E, et al: A systematic review of loneliness and common chronic physical conditions in adults. Open Psychol J 2015;8:113-132.

20 Holt-Lunstad J, Smith TB, Baker M, et al. Loneliness and social isolation as risk factors for mortality: a meta-analytic review. Perspect Psychol Sci 2015;10:227-237.

-21 VanderWeele TJ, Hawkley LC, Cacioppo JT: On the reciprocal association between loneliness and subjective well-being. Am J Epidemiol 2012;176:777-784.

22 The Norwegian Association of the Blind and Partially Sighted: Om Blindeforbundet, $w w w$. blindeforbundet.no/om-blindeforbundet (Zugriff 07.06.2018)
23 Lappegård T, Veenstra M: Life-course, generation and gender. LOGG 2007: field report of the Norwegian Generations and Gender Survey. Oslo, Statistics Norway, 2013, www.ssb.no/ en/befolkning/artikler-og-publikasjoner/lifecourse-generation-and-gender-logg-2007 (Zugriff 07.06.2018).

24 Hughes ME, Waite LJ, Hawkley LC, et al.: A short scale for measuring loneliness in large surveys: results from two population-based studies. Res Aging 2004;26:655-672.

25 Lasgaard M, Friis K, Shevlin M: «Where are all the lonely people?»: a population-based study of high-risk groups across the life span. Soc Psychiatry Psychiatr Epidemiol 2016;51: 1373-1384.

26 Cantril H: A study of aspirations. Sci Am 1963; 208:41-45.

27 Coxe S, West SG, Aiken LS: Generalized linear models; in: Little TD (Hrsg.) : The Oxford handbook of quantitative methods. New York, Oxford University Press, 2013, pp 26-52.

28 Knol MJ, VanderWeele TJ: Recommendations for presenting analyses of effect modification and interaction. Int J Epidemiol 2012;41: 514-520.

29 Qualter P, Vanhalst J, Harris R, et al.: Loneliness across the life span. Perspect Psychol Sci 2015;10:250-264.

30 World Health Organization: World report on disability. Genf, World Health Organization, 2011 www.who.int/disabilities/world_report/2011/en (Zugriff 07.06.2018).
31 McKee-Ryan F, Song Z, Wanberg CR, et al.: Psychological and physical well-being during unemployment: a meta-analytic study. J Appl Psychol 2005;90:53-76.

32 Hawker DS, Boulton MJ: Twenty years' research on peer victimization and psychosocial maladjustment: a meta-analytic review of cross-sectional studies. J Child Psychol Psychiatry 2000;41:441-455.

33 American Psychiatric Association: Diagnostic and statistical manual of mental disorders, fifth edition (DSM-5). Washington, American Psychiatric Pub, 2013.

34 Cacioppo JT, Hawkley LC: Perceived social isolation and cognition. Trends Cogn Sci 2009; 13:447-454.

35 Valtorta NK, Kanaan M, Gilbody S, et al.: Loneliness, social isolation and social relationships: what are we measuring? A novel framework for classifying and comparing tools. BMJ Open 2016;6:e010799.

36 Rothman KJ, Gallacher JEJ, Hatch EE: Why representativeness should be avoided. Int J Epidemiol 2013;42:1012-1014.

37 Statistics Norway. Statistikkbanken: Oslo: Statistics Norway, u.d., www.ssb.no/statistikkbanken (07.06.2018).

38 Schild S, Dalenberg CJ: Trauma exposure and traumatic symptoms in deaf adults. Psychol Trauma 2012;4:117-127. 